\title{
Neural Responses to BEGIN- and END-Stimuli of the Smoking Ritual in Nonsmokers, Nondeprived Smokers, and Deprived Smokers
}

\author{
Bastian Stippekohl*', Markus Winkler², Ronald F Mucha ${ }^{2}$, Paul Pauli ${ }^{2}$, Bertram Walter', Dieter Vaitl' and \\ Rudolf Stark'
}

'Bender Institute of Neuroimaging, University of Giessen, Germany; ${ }^{2}$ Department of Psychology, University of Würzburg, Germany

\begin{abstract}
Drug-associated stimuli (cues) have a prominent role in addiction research because they are able to provoke craving and relapses. Generally, drug cues are seen as conditioned excitatory stimuli, which elicit drug seeking and usage. However, newer data suggest differential effects for smoking stimuli depending on their stage in the smoking ritual. Specifically, stimuli associated with the terminal stage of smoke consumption (END-stimuli) may evoke reactivity opposite to the reactivity evoked by stimuli associated with the beginning of smoke consumption (BEGIN-stimuli). This fMRI study compared 20 nondeprived smokers with 20 nonsmokers to unravel the influence of smoking-related pictures displaying the beginning (BEGIN-stimuli) and termination (END-stimuli) of the smoking ritual on neural activity in the addiction network. In addition, 20 deprived smokers ( $12 \mathrm{~h}$ deprivation) were investigated to explore the effects of deprivation on the processing of these stimuli. In nondeprived smokers, BEGIN-stimuli reliably activated the addiction network (for example, the ventral striatum, orbitofrontal cortex, and anterior cingulate cortex (ACC)). In contrast, END-stimuli triggered a differential pattern of activations as well as deactivations; deactivations were found in the ventral striatum and the ACC. Deprivation had no clear effect on the responses triggered by BEGIN-stimuli, but affected the reactivity to END-stimuli. Our data clearly suggest that stimuli associated with different stages of the smoking ritual trigger differential neuronal responses. While BEGIN-stimuli generally seem to activate the addiction network, END-stimuli presumably have some inhibitory properties. This new finding might add to a more differentiated understanding of cue reactivity and addiction.

Neuropsychopharmacology (2010) 35, 1209-1225; do: I 0.1038/npp.2009.227; published online 20 January 2010
\end{abstract}

Keywords: addiction; smoking; cue reactivity; $\mathrm{fMRl}$; deprivation; terminal stimuli

\section{INTRODUCTION}

Core features of addiction are excessive craving, compulsive drug intake, and high rates of relapse (Self and Nestler, 1998; Lubman et al, 2004; Kalivas and Volkow, 2005). Current learning theories of addiction emphasize the prominent role of drug cues - stimuli associated with drug intake, which may induce craving, lapses, and relapses (Wikler, 1948; Stewart et al, 1984; Siegel, 1989; Robinson and Berridge, 1993, 2003). Empirical data support these assumptions for smokers and smoking cues (Niaura et al, 1988; O'Brien et al (1998); Carter and Tiffany, 1999).

Brain research confirmed that addiction is a disorder of learning and memory (Everitt et al, 2001; Hyman, 2005; Kauer and Malenka, 2007). Evidence suggests that

*Correspondence: B Stippekohl, Bender Institute of Neuroimaging, Justus-Liebig-University of Giessen, Otto-Behaghel-Str. 10H, Giessen, Hessen, 35394, Germany, Tel: + 49641992 6087, Fax: + 49641992 6309, E-mail: bastian.stippekoh|@psychol.uni-giessen.de

Received 17 August 2009; revised 30 November 2009; accepted 10 December 2009 drugs lead to pathological adaptations in a widely distributed neuronal network divided into four subcircuits: reward/salience (ventral tegmental area (VTA), ventral striatum), motivation/drive/craving (orbitofrontal cortex (OFC), insula), learning/memory (hippocampus, amygdala), and control (dorsolateral prefrontal cortex (DLPFC), anterior cingulate cortex (ACC)) (Volkow et al, 2003, 2004; Everitt and Robbins, 2005; Naqvi and Bechara, 2009). Smoking cues were found to activate this network in smokers but not in nonsmokers (Brody et al, 2002; Due et al, 2002; David et al, 2005; Smolka et al, 2006; Franklin et al, 2007).

However, stimuli associated with different stages of the smoking ritual seem to trigger differential responses. Using pictures of different stages of smoking, Mucha et al (1999, 2008) found evidence that pictures representing the beginning and the process of smoking (BEGIN-stimuli, for example, scenes of lighting a cigarette) elicit high craving. Pictures representing the terminal phase of smoking (ENDstimuli, for example, scenes of the disposal of a finished cigarette) elicit low craving, although they are associated 
with a high nicotine level in the body (see also Bushnell et al, 2000). A difference was also shown using a physiological measure of motivational state: contrary to END-stimuli, BEGIN-stimuli attenuate the startle response similar to positive pictures (Geier et al, 2000; Mucha et al, 2006; Mucha et al, 2008). However, END-stimuli are probably not simply weak cues as they are able to reduce the effects of BEGIN-stimuli when both are presented together (Mucha et al, 2008). Therefore, END-stimuli seem to trigger a unique reactivity, which even may oppose responses triggered by BEGIN-stimuli. Mucha et al (2008) suggested this to be an effect of the perceived smoke availability, which is high for BEGIN- and low for END-stimuli. Previous studies showed increased subjective, behavioral, physiological, and neuronal responses to smoking cues when smoking seems available (Carter and Tiffany, 2001; Wertz and Sayette, 2001; Wilson et al, 2004; McBride et al, 2006). Other studies showed that the end of drug consumption and stimuli predicting the nonoccurrence of the drug reduce drug seeking and wanting (Kearns et al, 2005; see also Panlilio et al, 2008). In sum, these results point to an inhibitory nature of END-stimuli.

There are, however, alternative explanations for the low cue reactivity of END-stimuli (Mucha et al, 2008). Therefore, it is important that research on END-stimuli has focused on subjective and physiological responses, but nothing is known about their neuronal representation. Accordingly, this fMRI study was designed to investigate whether END-stimuli have an impact on neuronal activity in the addiction network and whether they lead to inhibitory effects. Prototypical BEGIN- ('lighting a cigarette') and END-stimuli ('stubbing out a cigarette butt') were presented to nondeprived smokers, deprived smokers ( $12 \mathrm{~h}$ deprivation), and nonsmokers. Furthermore, our study included an expansion of the concept of BEGIN- and END-stimuli. Several animal conditioning studies suggest that conditioned stimuli (CS) lead to different behavioral responses depending on their temporal proximity to the unconditioned stimulus (UCS) (Silva et al, 1996, 1998; Silva and Timberlake, 1997; Timberlake, 1997). For example, with food as reinforcer, long CS-UCS intervals lead to general search behavior, whereas short intervals lead to focal search behavior. A similar functional organization may also be present in the smoking ritual. Thus, we added two exploratory stimuli ('taking a cigarette out of its box', 'the last puff) to represent a wider range of the smoking ritual.

Several directional hypotheses were put forward for BEGIN- and END-stimuli: for nondeprived and deprived smokers, we hypothesized that BEGIN-stimuli lead to neuronal activations, whereas END-stimuli should result in deactivations. Regarding group differences, we expected that these effects are significantly different between nondeprived smokers and nonsmokers. Similar group differences were expected between deprived and nondeprived smokers, because learning theories suggest an enhancement of the incentive value of drug-associated stimuli under deprivation (Stewart et al, 1984; Toates, 1994; Berridge, 2004). We had no directional hypotheses for the effects of the additional stimuli. However, we also expected significant group differences for them.

\section{MATERIALS AND METHODS}

\section{Subjects}

In all, 60 right-handed subjects participated in the study: 20 nondeprived smokers ( 11 female), 20 deprived smokers (10 female), and 20 nonsmokers (10 female). Nondeprived and deprived smokers were included if they had smoked for at least 3 years and a mean of at least 15 cigarettes per day in the last 12 months; nonsmokers had to have smoked $<10$ cigarettes in their lifetime. The Edinburgh Inventory of Handedness (Oldfield, 1971) ensured that only right-handed subjects participated. A self-generated questionnaire was used to assess general information regarding smoking history. The Fagerström Test of Nicotine Dependence (FTND; Schumann et al, 2003a) was used to assess the severity of addiction. Deprived smokers were instructed to go to bed before $2400 \mathrm{~h}$ on the day before the experiment and were scheduled after $1200 \mathrm{~h}$ on the day of the experiment. This resulted in a deprivation phase of at least $12 \mathrm{~h}$. The procedure was the same for nondeprived smokers and nonsmokers.

Most subjects were students receiving either money (10 euro/h) or course credit. No subject was taking regular medication or had a history of psychiatric or neurological illness. None of the participants reported abuse of other drugs. The study was performed in accordance with the ethical standards of the fifth revision of the Declaration of Helsinki and approved by the Ethical Committee of the German Psychological Association.

\section{Stimulus Material}

We used self-generated pictures and videos depicting different stages of the smoking ritual without showing many irrelevant or distracting features (for example, faces, surroundings, cigarette brands). As neutral controls, stimuli depicting equivalent temporal stages of teeth-brushing were used. Teeth-brushing can be matched to the smoking process with respect to temporal stages, body parts, and equipment. It can be considered as neutral because it is an overlearned everyday routine. All props (for example, ashtrays, lighters, toothbrushes, beakers) were matched for color.

We recorded 12 video clips using 12 different models (6 female) while smoking or teeth-brushing. We then cut four sequences ( $4 \mathrm{~s}$ each) out of each clip. Each represented one of four different stages of smoking or teeth-brushing. Then, one representative static picture from each clip was selected. Smoking stages were: 'taking a cigarette out of its box' (S1), 'lighting a cigarette' (S2), 'last puff on a cigarette' (S3), and 'stubbing out a cigarette butt' (S4). Teeth-brushing stages were: 'preparing a toothbrush with toothpaste' (T1), 'putting a toothbrush into ones mouth' (T2), 'taking a toothbrush out of ones mouth' (T3), and 'putting a toothbrush back into the beaker' (T4). Overall, eight different picture categories were used, with every smoking stage having its own teeth-brushing stage as control (S1, S2, S3, S4, T1, T2, T3, T4). Among these stimuli, we focused on S2 and S4, as they are prototypical BEGIN- (S2) and END-stimuli (S4) of smoking. S1 and S3 were considered as exploratory to account for a wider range of the smoking ritual.

To study differential effects of pictures and video clips, both were presented in separate and counterbalanced 
sessions on different days 1 week apart. The data regarding the picture stimuli will be the focus of this paper. Results regarding the video stimuli will be reported separately. All pictures were presented in $800 \times 600$ pixel resolution. An LCD projector (model EPSON EMP-7250) projected them onto a screen at the end of the scanner (visual field $=18^{\circ}$ ) where they were viewed through a mirror mounted on the head coil. Pictures were presented for $4 \mathrm{~s}$ in randomized order (restriction: not more than two stimuli of the same category after one another).

Participants rated the stimuli right after their presentation on the dimensions craving, valence, and arousal, on 9-point Likert scales using a three-button keypad (left, right, enter). For craving, the scale was visualized with bars of different heights representing the amount of craving. For valence and arousal, a computerized version of the Self Assessment Manikin (SAM; Bradley and Lang, 1994) was applied. Ratings of craving were assessed after each stimulus. Valence and arousal ratings interchanged to keep the experiment short (each stimulus category was rated half on valence and half on arousal).

\section{Procedure}

Subjects participated in three different sessions. Session 1: The study was explained, possible contraindications were checked, and written consent was obtained. Smokers were randomly assigned to the nondeprived or the deprived group. All subjects were instructed to go to bed before $2400 \mathrm{~h}$ on the days before the experiments (sessions 2 and 3). Deprived smokers were instructed not to smoke from bedtime to the end of the experimental sessions. After reading the instructions, all smokers were told to smoke one cigarette. This was necessary to measure a carbon monoxide (CO) and craving baseline (German version of the Questionnaire on Smoking Urges, QSU-G; Müller et al, 2001) when nondeprived. $\mathrm{CO}$ values and craving were also assessed in nonsmokers. All subjects had to fill in a selfgenerated questionnaire regarding their individual smoking history. Thereafter, an anatomical measurement took place in the MRI scanner to get the participants accustomed. Afterwards, smokers completed different smoking-related questionnaires (analyses not included).

Session 2 and 3: Each experimental session started between noon and $1800 \mathrm{~h}$. Nondeprived smokers were allowed to smoke one cigarette before the procedure started. Then, all subjects completed a CO measurement. Subsequently, they filled in the German version of the Questionnaire on Smoking Urges. CO levels were used to confirm deprivation. All deprived smokers had CO levels $\leqslant 13$ CO p.p.m., which is similar to the mean value found in other studies with overnight deprivation (Della Casa et al, 1999; Mucha et al, 1999; Due et al, 2002). Next, participants were familiarized with the stimulus presentation and rating procedure outside the scanner. Only neutral pictures from the International Affective Picture System (IAPS; Lang et al, 1995) were presented. Subjects were then placed in the scanner. Another test rating took place before the experiment. After the experiment, all smokers were allowed to smoke. They then filled in several personality questionnaires (analyses not included). After completing all sessions, participants were paid and debriefed.

\section{Data Acquisition and Analysis}

Brain images were acquired using a $1.5 \mathrm{~T}$ whole-body tomograph (Siemens Symphony) with a standard head coil. Structural image acquisition consisted of 160 T1-weighted sagittal images (MPRage, 1-mm slice thickness). A gradient echo field map sequence was measured before the functional run to get information for unwarping B0 distortions. For functional imaging a total of 780 volumes were registered using a $\mathrm{T} 2{ }^{*}$-weighted gradient echo-planar imaging sequence with 25 transversal slices covering the whole brain (slice thickness $=5 \mathrm{~mm} ; 1 \mathrm{~mm}$ gap; descending; $\mathrm{TR}=2.5 \mathrm{~s} ; \mathrm{TE}=55 \mathrm{~ms}$; flip angle $=90^{\circ}$; field of view $=$ $192 \times 192 \mathrm{~mm}$; matrix size $=64 \times 64)$. The first three volumes were discarded because of an incomplete steady state of magnetization. Orientation of slices was parallel to the AC-PC line.

The statistical parametric mapping software package (SPM5, Wellcome Department of Cognitive Neurology, London, UK) implemented in Matlab (Mathworks, Natick, MA, Release 2007b) was used for preprocessing and statistical analyses. Origin coordinates were adjusted to the anterior commissure (AC), and realignment (thirdorder B-spline) and unwarping, slice time correction, and normalization to the standard brain of the Montreal Neurological Institute were performed. Smoothing was executed with an isotropic three-dimensional Gaussian filter with a full width at half maximum (FWHM) of $9 \mathrm{~mm}$.

For the event-related design, each picture presentation was modeled with a boxcar function convolved with a hemodynamic response function (duration $4 \mathrm{~s}$ ) in the General Linear Model of SPM. The six movement parameters of the rigid body transformation applied by the realignment procedure were introduced as covariates in the model. The serial correlation in the voxel-based time series was considered as a first-order autoregressive process. A high-pass filter (time constant $=128 \mathrm{~s}$ ) was implemented by using cosine functions in the design matrix.

For analyses of hemodynamic responses, contrasts between smoking and corresponding control stimuli (smoking minus control) were calculated on the first level: S1-T1 is called TAKING OUT, S2-T2 BEGIN, S3-T3 LAST PUFF, and S4-T4 END. These contrasts were then used as dependent variables on the second level. Activations were defined as positive differences between smoking and control stimuli; deactivations as negative differences. The second-level model was a variance analytical model with the factors group and stage (four stages). Our data analysis comprised a confirmatory part and an exploratory part: in the confirmatory part, appropriate contrasts for each hypothesis were constructed within this model and $t$-tests were applied to these contrasts. Group comparisons (nondeprived smokers vs nonsmokers; deprived smokers $v s$ nondeprived smokers) were carried out for (1) BEGIN, (2) END, and (3) differences between BEGIN and END. Afterwards, exploratory analyses were performed by comparing the groups (nondeprived smokers $v s$ nonsmokers; deprived smokers $v s$ nondeprived smokers) for all four contrasts in two $2 \times 4$ plans.

Regions of interest (ROI) analyses were performed for the DLPFC, OFC, ACC, VTA, ventral striatum, amygdala, 
hippocampus, and insula. Results were corrected for multiple testing in each ROI according to Worsley (2001).

Ratings were analyzed with SPSS 17.0. The analysis was analogous to that of the brain data but without computing differences between smoking and control stimuli beforehand. For the repeated measure factor stage, the Greenhouse-Geisser correction was applied. Post hoc comparisons were performed using the Bonferroni adjustment for multiple comparisons. For all statistical analyses, $\alpha$ was set to 0.05 .

\section{RESULTS}

\section{Group Characteristics}

The final analyses are based on 20 deprived smokers (10 female), 19 nondeprived smokers (11 female), and

Table I Mean (SE) of Age, Years Smoked, Cigarettes/Day in the Last 12 Months, Scores on the Fagerström Test of Nicotine Dependence (FTND), the Questionnaire of Smoking Urges (QSU), and carbon monoxide (CO) Measures

\begin{tabular}{lccr}
\hline & $\begin{array}{c}\text { Nonsmokers } \\
(\mathbf{n}=\mathbf{I 7} \mathbf{9 f})\end{array}$ & $\begin{array}{c}\text { Nondeprived } \\
\text { smokers } \\
(\mathbf{n}=\mathbf{I 9 ;} \mathbf{I I f})\end{array}$ & $\begin{array}{c}\text { Deprived } \\
\text { smokers } \\
(\mathbf{n}=\mathbf{2 0} ; \mathbf{~ I 0 f})\end{array}$ \\
\hline Age (years) & $24.59(0.79)^{\mathrm{a}}$ & $27.21(1.37)^{\mathrm{a}}$ & $25.80(1.68)^{\mathrm{a}}$ \\
Years smoked & - & $10.77(1.17)^{\mathrm{a}}$ & $10.29(1.75)^{\mathrm{a}}$ \\
Cigarettes/day & - & $20.79(0.83)^{\mathrm{a}}$ & $19.43(1.33)^{\mathrm{a}}$ \\
FTND & - & $4.58(0.49)^{\mathrm{a}}$ & $3.95(0.39)^{\mathrm{a}}$ \\
QSU baseline & $1.11(0.03)^{\mathrm{a}}$ & $2.44(0.19)^{\mathrm{b}}$ & $2.82(0.20)^{\mathrm{b}}$ \\
QSU prescan & $1.07(0.35)^{\mathrm{a}}$ & $2.34(0.20)^{\mathrm{b}}$ & $4.78(0.24)^{\mathrm{c}}$ \\
CO baseline & $2.59(0.23)^{\mathrm{a}}$ & $28.32(2.69)^{\mathrm{b}}$ & $28.25(2.68)^{\mathrm{b}}$ \\
CO prescan & $2.59(0.24)^{\mathrm{a}}$ & $28.53(2.56)^{\mathrm{b}}$ & $6.60(0.56)^{\mathrm{c}}$ \\
\hline
\end{tabular}

Groups with same indices did not differ significantly.
17 nonsmokers (9 female). Four subjects had to be excluded: one smoker and one nonsmoker because of technical problems and two nonsmokers because they had smoked more than 10 cigarettes in their lifetime. Table 1 summarizes relevant group characteristics.

As intended, the groups did not differ in age. Nondeprived smokers and deprived smokers did not differ in years smoked, mean number of cigarettes per day within the last 12 months, and FTND score. As expected, the three groups differed in craving and $\mathrm{CO}$ levels at baseline measured in session 1 (QSU baseline: $\mathrm{F}(2,52)=26.261$, $p<0.001$; CO baseline: $\mathrm{F}(2,53)=39.694, p<0.001)$. Craving scores and CO levels were higher in nondeprived smokers and deprived smokers than in nonsmokers (QSU baseline: nondeprived smokers $v s$ nonsmokers: mean difference $=1.33, p<0.001$, deprived smokers $v s$ nonsmokers: mean difference $=1.71, p<0.001$; CO baseline: nondeprived smokers $v s$ nonsmokers: mean difference $=25.73$, $p<0.001$; deprived smokers $v s$ nonsmokers: mean difference $=25.66, p<0.001)$. On the day of the scanning session, deprived smokers showed higher craving scores $(t(37)=7.740, p<0.001)$ and lower CO levels $(t(37)=-8.585, \quad p<0.001)$ than nondeprived smokers. They also had significantly higher craving scores and lower CO levels than at baseline (QSU: $t(19)=7.976$, $p<0.001$; CO levels: $t(19)=-8.386, p<0.001)$. Thus, CO and QSU levels confirmed the effectiveness of the deprivation manipulation.

\section{Picture Ratings for BEGIN- and END-Stimuli}

The following section focuses on the rating data for smoking stimuli (differences to control stimuli, differences to other smoking stimuli, group differences). Differences with regard to control stimuli (differences to other control stimuli, group differences) are not reported in the text but can be seen in Table 2 .

Table 2 Mean (SE) of Craving, Valence, and Arousal Ratings (Range for All Three Scales: I-9) for Smoking (SI, S2, S3, S4), and Control Stimuli (TI, T2, T3, T4) for Nonsmokers, Nondeprived Smokers, and Deprived Smokers

\begin{tabular}{|c|c|c|c|c|c|c|c|c|}
\hline & SI & $\mathbf{S 2}$ & $\mathbf{S 3}$ & S4 & TI & T2 & T3 & T4 \\
\hline \multicolumn{9}{|l|}{ Nonsmokers } \\
\hline Craving & $1.00(0.00)$ & $1.00(0.00)$ & $1.00(0.00)$ & $1.00(0.00)$ & $1.00(0.00)$ & $1.00(0.00)$ & $1.00(0.00)$ & $1.00(0.00)$ \\
\hline Valence & $4.40(0.40)$ & $4.22(0.45)$ & $4.03(0.45)$ & $4.06(0.46)$ & $5.92(0.29)$ & $5.64(0.31)$ & $5.70(0.33)$ & $5.87(0.28)$ \\
\hline \multicolumn{9}{|c|}{ Nondeprived smokers } \\
\hline Craving & $4.57(0.52)$ & $4.70(0.53)$ & $4.05(0.42)$ & $3.31(0.35)$ & $2.32(0.32)$ & $2.40(0.35)$ & $2.35(0.34)$ & $2.36(0.32)$ \\
\hline Valence & $6.06(0.20)$ & $6.00(0.22)$ & $5.50(0.25)$ & $5.08(0.32)$ & $5.9 \mid(0.22)$ & $5.72(0.30)$ & $5.73(0.28)$ & $5.98(0.25)$ \\
\hline Arousal & $3.77(0.33)$ & $3.85(0.35)$ & $3.71(0.30)$ & $3.44(0.32)$ & $3.09(0.32)$ & $3.11(0.31)$ & $3.40(0.36)$ & $3.05(0.31)$ \\
\hline Arousal & $6.01(0.27)$ & $6.25(0.25)$ & $5.87(0.28)$ & $5.18(0.28)$ & $4.10(0.37)$ & $4.27(0.38)$ & $4.14(0.40)$ & $3.90(0.32)$ \\
\hline
\end{tabular}

S2 and S4 (respectively T2 and T4) are highlighted because we mainly focus on BEGIN-(S2) and END-(S4) stimuli. 


\section{Nondeprived Smokers Compared with Nonsmokers}

Craving: In nondeprived smokers, BEGIN- and END-stimuli led to more craving than the corresponding control stimuli (BEGIN: $t(18)=4.40, \quad p<0.001 ; \quad$ END: $t(18)=2.57$, $p=0.019)$ (Figure 1, Table 2). BEGIN-stimuli led to higher craving than END-stimuli $(t(18)=4.22 ; p=0.001)$. For nonsmokers, no significant results were found. Comparing both groups, nondeprived smokers revealed higher craving ratings than nonsmokers for BEGIN- and END-stimuli (BEGIN: $t(18)=6.96, p<0.001$; END: $t(18)=6.63, p<0.001$ ).

Valence: Nondeprived smokers rated END-stimuli as significantly less pleasurable than the corresponding control stimuli $(t(18)=-3.39, p=0.003$ ) (Table 2). BEGIN-stimuli were rated as more pleasurable than END-stimuli $(t(18)=2.69, p=0.015)$. Nonsmokers rated BEGIN- and END-stimuli significantly less pleasurable than the corresponding control stimuli (BEGIN: $t(16)=-2.49, p=0.024$; END: $t(16)=-3.21, p=0.005)$. Comparing both groups, nondeprived smokers rated BEGIN-stimuli as significantly more pleasurable than nonsmokers $(t(23.09)=3.57, p=0.002)$.

Arousal: Nondeprived smokers rated BEGIN-stimuli as significantly more arousing than the corresponding control stimuli $(t(18)=3.65, p=0.002)$ (Table 2$)$. The same was true for nonsmokers $(t(16)=2.17, p=0.046)$. Comparing both groups, no significant differences were observed.

\section{Deprived Smokers Compared with Nondeprived Smokers}

The ratings of one deprived smoker had to be excluded from the analysis due to excessive missing values.

Craving: In deprived smokers, BEGIN- and END-stimuli led to more craving than the corresponding control stimuli (BEGIN: $t(18)=5.96, p<0.001$; END: $t(18)=4.88$, $p<0.001$ ) (Figure 1, Table 2). BEGIN-stimuli led to higher craving than END-stimuli $(t(18)=4.38 ; p<0.001)$.
In comparison with nondeprived smokers, deprived smokers showed higher craving ratings for BEGINand END-stimuli (BEGIN: $t(36)=2.86, p=0.007$; END: $t(36)=3.71, p=0.001)$.

Valence: Deprived smokers rated BEGIN-stimuli as significantly more pleasurable than the corresponding control stimuli $(t(18)=3.07, p=0.007)$ and END-stimuli $(t(18)=2.87, p=0.010)$ (Table 2). In comparison with nondeprived smokers, no significant differences were observed.

Arousal: Deprived smokers rated BEGIN- and ENDstimuli as significantly more arousing than the corresponding control stimuli (BEGIN: $t(18)=4.34, p<0.001$; END: $t(18)=4.48, p<0.001) \quad($ Table 2$)$. BEGIN-stimuli were rated as more arousing than END-stimuli $(t(18)=4.82$, $p<0.001)$. In comparison with nondeprived smokers, deprived smokers rated BEGIN- as well as END-stimuli as significantly more arousing (BEGIN: $t(36)=5.58, p<0.001$; END: $t(36)=4.08, p<0.001)$.

\section{Ratings of Additional Stimuli}

Craving: 'Taking a cigarette out of its box' led to higher craving in nondeprived smokers and deprived smokers than the corresponding control stimuli (nondeprived smokers: $t(18)=4.43, \quad p<0.001$; deprived smokers: $t(18)=5.99$, $p<0.001$ ) (Table 2). No such differences were found for nonsmokers. Similar results were found for 'the last puff' (nondeprived smokers: $t(18)=4.00, p=0.001$; deprived smokers: 5.60, $p<0.001$ ).

Valence: Deprived smokers rated 'taking a cigarette out of its box' as significantly more pleasurable than the corresponding control stimuli $(t(18)=3.64, p=0.002)$ (Table 2). Nonsmokers rated this stimulus as significantly less pleasurable than control stimuli $(t(16)=-2.72$, $p=0.015)$. No significant results were found for nondeprived smokers. Similar results were found for 'the last puff

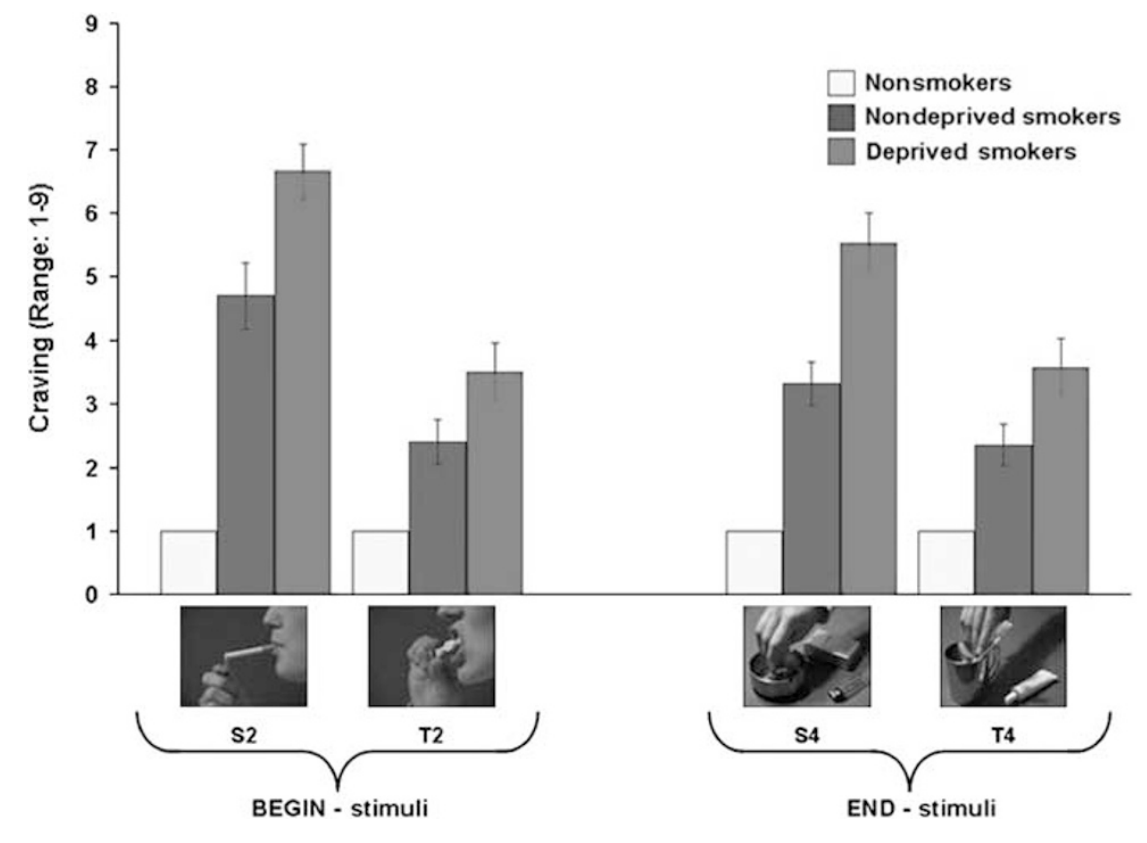

Figure I Craving ratings (scale range: 1-9) for BEGIN- and END-stimuli. 
(deprived smokers: $t(18)=3.54, p=0.002$; nonsmokers: $t(16)=-3.09, p=0.007)$.

Arousal: 'Taking a cigarette out of its box' was rated significantly more arousing than the corresponding control stimuli by nondeprived smokers and deprived smokers (nondeprived smokers: $t(18)=4.62, p<0.001$; deprived smokers: $t(18)=4.49, p<0.001$ ) (Table 2). No significant results were found for nonsmokers. 'The last puff was rated as significantly more arousing than the corresponding control stimuli by deprived smokers $(t(18)=4.41$, $p<0.001)$.

\section{Exploratory Analyses of Differences Between the Four Smoking Stimuli}

To account for group differences in changes over time, the interaction between group and stage was analyzed in two $2 \times 4$ plans.

Craving: Comparing nondeprived smokers and nonsmokers, significant main effects of group and stage as well as a significant group $\times$ stage interaction were found (for all F-values $p<0.001)$. Post hoc analyses revealed that for nondeprived smokers, END-stimuli led to significantly lower craving than all other smoking stimuli (all $p<0.01$ ). BEGIN-stimuli led to significantly more craving than 'the last puff (mean difference $=0.65, p=0.045$ ), whereas no significant differences were found between BEGIN-stimuli and 'taking a cigarette out of its box'.

Comparing deprived smokers and nondeprived smokers, significant main effects of group and stage were found, with deprived smokers showing higher craving (all $p<0.004)$. Post hoc analyses in both groups combined revealed that END-stimuli led to significantly lower craving than all other smoking stimuli (all $p<0.001$ ). BEGIN-stimuli led to significantly more craving than 'taking a cigarette out of its box' (mean difference $=0.25, p=0.038$ ) and 'the last puff (mean difference $=0.58, p=0.007$ ), whereas no significant differences were found between 'taking a cigarette out of its box' and 'the last puff'.

Valence: Comparing nondeprived smokers and nonsmokers, significant main effects of group and stage were found, with nondeprived smokers showing higher ratings (all $p<0.003$ ) (Table 2). Post hoc analyses in both groups combined revealed that 'taking a cigarette out of its box' was rated as significantly more pleasurable than 'the last puff and END-stimuli. BEGIN-stimuli were rated as significantly more pleasurable than 'the last puff' (all $p<0.05$ ).

Comparing deprived smokers and nondeprived smokers, a significant main effect of stage was found $(\mathrm{F}(1.90$, $68.33)=11.39, p<0.001)$. Post hoc analyses in both groups combined revealed that END-stimuli were rated as significantly less pleasurable than all other smoking stimuli (all $p<0.01)$.

Arousal: Comparing nondeprived smokers and nonsmokers, no significant results were found (Table 2).

Comparing deprived smokers and nondeprived smokers, significant main effects of group and stage were found, with deprived smokers showing higher ratings (all $p<0.002$ ). Post hoc analyses in both groups combined revealed that END-stimuli were rated as significantly less arousing than all other smoking stimuli (all $p<0.05$ ).

\section{Brain Responses to BEGIN- and END-Stimuli}

Nondeprived smokers compared with nonsmokers

$B E G I N$ : For the contrast BEGIN, nondeprived smokers showed activations in the VTA, ventral striatum, OFC, ACC, DLPFC, hippocampus, and the insula (Table 3). In nonsmokers, we found activations in the OFC, ACC, and in the DLPFC. Comparing both groups, nondeprived smokers exhibited significantly greater activations than nonsmokers in the VTA, DLPFC, and insula (Table 4).

END: For the contrast END, nondeprived smokers showed deactivations in the ventral striatum, ACC, DLPFC, and the insula (Table 3). Yet despite deactivations, they also showed activations in the OFC, DLPFC, hippocampus, and the insula. No activations or deactivations were found for nonsmokers. Comparing both groups, nondeprived smokers showed stronger activations than nonsmokers in the OFC and the DLPFC (Table 4).

Differences between the contrasts BEGIN and END (BEGIN-END): Nondeprived smokers showed stronger neuronal activity for BEGIN than for END in the ventral striatum and ACC (Table 5). Nonsmokers showed no differences between these contrasts. Comparing both groups, nondeprived smokers revealed smaller differences between BEGIN and END in the OFC than nonsmokers (Table 4).

Figure 2 visualizes the most important findings. 'Lighting up a cigarette', a prototypical BEGIN-stimulus, led to activations in the ACC, DLPFC, and the ventral striatum of nondeprived smokers. In contrast, 'stubbing out a cigarette butt', a prototypical END-stimulus, triggered deactivations in exactly the same structures.

\section{Deprived Smokers Compared with Nondeprived Smokers}

$B E G I N$ : For the contrast BEGIN, deprived smokers showed activations in the VTA, OFC, ACC, DLPFC, hippocampus, and the insula (Table 3 ). In comparison with nondeprived smokers, deprived smokers showed a significantly stronger activation in the DLPFC (Table 4). However, at a different voxel coordinate, nondeprived smokers also showed a significantly stronger activation than deprived smokers in this structure.

END: For the contrast END, deprived smokers showed deactivations in the OFC and DLPFC. They also showed activations in the OFC and ventral striatum (Table 3). In comparison with nondeprived smokers, an effect of deprivation was found in the OFC, DLPFC, and insula, with deprived smokers showing significantly lower neuronal activity than nondeprived smokers (Table 4).

Differences between the contrasts BEGIN and END: In deprived smokers, stronger neuronal activity for BEGIN than for END was found in the VTA, OFC, ACC, DLPFC, and the insula (Table 5). In comparison with nondeprived smokers, deprived smokers showed significantly greater differences in the OFC and DLPFC (Table 4).

\section{Brain Responses to Additional Smoking Stimuli (TAKING OUT, LAST PUFF)}

For the contrast TAKING OUT, nondeprived smokers showed activations in the OFC, ACC, DLPFC, and the insula (Table 3 ). In addition, they showed deactivations in 
Table 3 Significant Activations and Deactivations for the Contrasts TAKING OUT, BEGIN, LAST PUFF, and END in Nonsmokers, Nondeprived Smokers, and Deprived Smokers

\begin{tabular}{llllllllll}
\hline Contrast & Structures & Side & $x$ & $y$ & $z$ & $\boldsymbol{t}_{\max }$ & score & Cluster size & $\boldsymbol{P}_{\text {corr }}$ \\
\hline Nonsmokers & & & &
\end{tabular}

No significant results

BEGIN

Activations

$\begin{array}{lrrrrrrr}\text { Orbitofrontal cortex } & \mathrm{L} & 0 & 54 & -15 & 4.29 & 292 & 0.009 \\ \text { Orbitofrontal cortex } & \mathrm{R} & 3 & 54 & -18 & 4.22 & 180 & 0.011 \\ \text { Anterior cingulate cortex } & \mathrm{R} & 6 & 45 & 27 & 3.47 & 138 & 0.034 \\ \text { Dorsolateral prefrontal cortex } & \mathrm{R} & 18 & 45 & 36 & 4.29 & 54 & 0.015\end{array}$

Deactivations

No significant results

LAST PUFF

No significant results

END

No significant results

Nondeprived smokers

TAKING OUT

Activations

Orbitofrontal cortex

Anterior cingulate cortex

Anterior cingulate cortex

Dorsolateral prefrontal cortex

Dorsolateral prefrontal cortex

Insular cortex

Insular cortex

Deactivations

Ventral striatum

Anterior cingulate cortex

Dorsolateral prefrontal cortex

BEGIN

Activations

Ventral tegmental area

Ventral striatum

Orbitofrontal cortex

Orbitofrontal cortex

Anterior cingulate cortex

Anterior cingulate cortex

Dorsolateral prefrontal cortex

Dorsolateral prefrontal cortex

Hippocampus

Insular cortex

Insular cortex

Deactivations

No significant results

LAST PUFF

Activations

$\begin{array}{rrrr}-42 & 18 & -9 & 5.40 \\ 3 & 21 & 21 & 3.69 \\ 6 & 18 & 24 & 3.82 \\ -33 & 3 & 54 & 5.90 \\ 27 & 0 & 57 & 4.64 \\ -39 & 15 & -6 & 6.43 \\ 33 & 24 & 3 & 4.88\end{array}$

15


Neural responses to BEGIN- and END-stimuli

B Stippekohl et al

Table 3 Continued

\begin{tabular}{|c|c|c|c|c|c|c|c|c|}
\hline Contrast & Structures & Side & $x$ & $y$ & $\mathbf{z}$ & $t_{\max }$ score & Cluster size & Pcorr \\
\hline & Ventral striatum & L & -15 & 6 & -12 & 3.64 & 22 & 0.007 \\
\hline & Ventral striatum & $\mathrm{R}$ & 15 & 9 & 0 & 3.38 & 91 & 0.017 \\
\hline & Orbitofrontal cortex & $\mathrm{R}$ & 36 & 30 & -15 & 5.15 & 861 & $<0.001$ \\
\hline & Anterior cingulate cortex & $\mathrm{L}$ & -6 & 30 & 30 & 6.61 & 332 & $<0.001$ \\
\hline & Dorsolateral prefrontal cortex & $\mathrm{R}$ & 42 & 18 & 39 & 6.26 & 2231 & $<0.001$ \\
\hline & Amygdala & $L$ & -30 & 0 & -27 & 5.85 & 69 & $<0.001$ \\
\hline & Amygdala & $\mathrm{R}$ & 36 & 0 & -24 & 5.34 & 80 & $<0.001$ \\
\hline & Hippocampus & $L$ & -24 & -27 & -21 & 5.33 & 465 & $<0.001$ \\
\hline & Hippocampus & $\mathrm{R}$ & 33 & -15 & -12 & 6.17 & 495 & $<0.001$ \\
\hline
\end{tabular}

Deactivations

No significant results

END

Activations

Orbitofrontal cortex

Orbitofrontal cortex
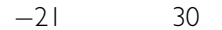

$-12$

6.72

4.05

4.39

Dorsolateral prefrontal cortex

Dorsolateral prefrontal cortex

Hippocampus

4.71

Insular cortex

Deactivations

Ventral striatum

Anterior cingulate cortex

Anterior cingulate cortex

Dorsolateral prefrontal cortex Insular cortex

$$
33
$$$$
-15
$$

Deprived smokers

TAKING OUT

Activations

Deactivations

\section{Orbitofrontal cortex}

Orbitofrontal cortex

Anterior cingulate cortex

Anterior cingulate cortex

Dorsolateral prefrontal cortex

Dorsolateral prefrontal cortex

Hippocampus

Hippocampus

Insular cortex

Insular cortex

No significant results

-12
24
-9
3
-15
33
-12
15
-45
48

\begin{abstract}
51
\end{abstract}

$-3$

5.15

680

429

306

84

859

260

152

316

303

367

$<0.00$ I

0.005

$<0.001$

0.001

$<0.00$ I

0.023

$<0.00$ I

0.004

$<0.00$ I

0.001

BEGIN

Activations 
Table 3 Continued

\begin{tabular}{|c|c|c|c|c|c|c|c|c|}
\hline Contrast & Structures & Side & $x$ & $y$ & $\mathbf{z}$ & $t_{\max }$ score & Cluster size & Pcorr \\
\hline & Anterior cingulate cortex & L & -9 & 48 & -3 & 3.68 & 113 & 0.021 \\
\hline & Dorsolateral prefrontal cortex & L & -21 & -3 & 66 & 4.31 & 1015 & 0.013 \\
\hline & Dorsolateral prefrontal cortex & $\mathrm{R}$ & 36 & -9 & 57 & 4.43 & 197 & 0.009 \\
\hline & Hippocampus & $L$ & -30 & -21 & -27 & 3.92 & $|5|$ & 0.012 \\
\hline & Hippocampus & $\mathrm{R}$ & 18 & -3 & -27 & 3.79 & 25 & 0.020 \\
\hline
\end{tabular}

Deactivations

No significant results

LAST PUFF

Activations

Ventral striatum
Ventral striatum
Orbitofrontal cortex
Anterior cingulate cortex
Dorsolateral prefrontal cortex
Dorsolateral prefrontal cortex
Amygdala
Hippocampus
Hippocampus
Insular cortex
Insular cortex

$\begin{array}{lr}\mathrm{L} & -3 \\ \mathrm{R} & -36 \\ \mathrm{~L} & -6 \\ \mathrm{~L} & -42 \\ \mathrm{~L} & 39 \\ \mathrm{R} & -18 \\ \mathrm{R} & 21 \\ \mathrm{~L} & -42 \\ \mathrm{R} & \\ \mathrm{L} & 39 \\ \mathrm{R} & \end{array}$

-3
6
-36
-6
-42
39
24
-18
21
-42
39

12
3
42
48
48
45
-9
-6
-6
3
0

-6
-9
-9
6
6
3
-15
-33
-33
9
3

3.12
3.26
3.91
3.54
4.29
4.48
3.02
3.71
4.01
4.12
4.02

77
63
227
207
755
354
41
211
287
305
191

Deactivations

No significant results

END

Activations

\section{Orbitofrontal cortex \\ Orbitofrontal cortex}

Ventral striatum

$\mathrm{L}$
$\mathrm{L}$

-9
3
-3

3.80
3.97
4.24

3.80
3.97
4.24
0.041

0.026

0.001

\section{Deactivations}

Orbitofrontal cortex
Dorsolateral prefrontal cortex

Dorsolateral prefrontal cortex

\begin{tabular}{lr}
$R$ & 27 \\
$L$ & -18 \\
$R$ & 24 \\
\hline
\end{tabular}

\begin{tabular}{rrrrr}
54 & -3 & 4.14 & 125 & 0.015 \\
6 & 66 & 3.91 & 206 & 0.049 \\
51 & 0 & 4.58 & 648 & 0.005 \\
\hline
\end{tabular}

the ventral striatum, ACC, and DLPFC. Deprived smokers showed activations in the OFC, ACC, DLPFC, hippocampus, and the insula (Table 3). No activations or deactivations were found for nonsmokers.

For the contrast LAST PUFF, nondeprived smokers showed activations in the VTA, ventral striatum, OFC, ACC, DLPFC, amygdala, hippocampus, and the insula (Table 3). Deprived smokers showed activations in the ventral striatum, OFC, ACC, DLPFC, amygdala, hippocampus, and the insula (Table 3 ). No activations or deactivations were found for nonsmokers.

\section{Exploratory Analysis of Differences Between the Four Smoking Stimuli}

To account for group differences in changes over time, the interaction between group and stage was analyzed in two $2 \times 4$ plans.
Comparing nondeprived smokers and nonsmokers, a significant interaction was found in the right amygdala (Table 6); the greatest differences between both groups appeared for BEGIN and LAST PUFF. Nondeprived smokers showed strong amygdala activations for LAST PUFF and deactivations for BEGIN; the reverse was true for nonsmokers. Contrast values for TAKING out and END were close to zero in both groups.

Comparing deprived smokers and nondeprived smokers, a significant main effect of stage was found in the OFC, ACC, and the insula. Figure 3 shows the contrast estimates for the combined group (nondeprived and deprived smokers) and the four conditions for the ACC. It is noteworthy that contrast values decreased over the time course of the smoking ritual and showed deactivations for END. Similar progressions were found for the OFC and the insula.

In addition to this main effect, we found significant interactions between group and stage in the DLPFC and hippocampus (Table 6). Figure 4 shows the DLPFC- 
Table 4 Group Differences for the Contrasts BEGIN, END, and BEGIN-END

\begin{tabular}{|c|c|c|c|c|c|c|c|c|}
\hline Contrast & Structures & Side & $x$ & $y$ & $\mathbf{z}$ & $t_{\max }$ score & Cluster size & Pcorr \\
\hline \multicolumn{9}{|c|}{ Nondeprived smokers-nonsmokers } \\
\hline \multicolumn{9}{|c|}{ BEGIN } \\
\hline & Ventral tegmental area & & 0 & -18 & -6 & 2.74 & 11 & 0.024 \\
\hline & Dorsolateral prefrontal cortex & L & -39 & 48 & 18 & 3.95 & 205 & 0.043 \\
\hline & Insular cortex & L & -36 & 6 & -6 & 3.61 & 135 & 0.033 \\
\hline & Orbitofrontal cortex & L & -18 & 45 & -12 & 4.86 & 283 & 0.001 \\
\hline & Dorsolateral prefrontal cortex & L & -24 & -6 & 60 & 4.28 & 242 & 0.014 \\
\hline & Dorsolateral prefrontal cortex & $\mathrm{R}$ & 27 & 6 & 45 & 5.43 & 629 & $<0.001$ \\
\hline
\end{tabular}

BEGIN-END

No significant results

Nonsmokers-nondeprived smokers

BEGIN

No significant results

END

No significant results

BEGIN-END

Orbitofrontal cortex

$-21$

48

$-15$

3.84

144

0.036

Deprived smokers-nondeprived smokers

BEGIN

Dorsolateral prefrontal cortex

$-21$

30

60

4.46

591

0.008

END

BEGIN-END

No significant results

Orbitofrontal cortex

Dorsolateral prefrontal cortex

$L \quad-18$

48

$$
-12
$$

3.80

144

445

0.041

0.029

Nondeprived smokers-deprived smokers

BEGIN

END

Dorsolateral prefrontal cortex

$-18$

48

21

4.02

290

0.035

Orbitofrontal cortex

Orbitofrontal cortex

Dorsolateral prefrontal cortex

Dorsolateral prefrontal cortex

Insular cortex

$\begin{array}{lr}\mathrm{L} & -18 \\ \mathrm{R} & 27 \\ \mathrm{~L} & -24 \\ \mathrm{R} & 18 \\ \mathrm{~L} & -30\end{array}$

BEGIN-END

No significant results

48
39
-3
3
21

-12
-12
63
66
12

4.28

3.80

4.19

4.12

3.54
0.043

14

4


Table 5 Comparison of the Contrasts BEGIN and END in Nonsmokers, Nondeprived Smokers, and Deprived Smokers

\begin{tabular}{|c|c|c|c|c|c|c|c|c|}
\hline Contrast & Structures & Side & $x$ & $y$ & $\mathbf{z}$ & $t_{\max }$ score & Cluster size & Pcorr \\
\hline \multicolumn{9}{|l|}{ Nonsmokers } \\
\hline \multicolumn{9}{|l|}{ BEGIN-END } \\
\hline & No significant results & & & & & & & \\
\hline \multicolumn{9}{|l|}{ END-BEGIN } \\
\hline & No significant results & & & & & & & \\
\hline \multicolumn{9}{|c|}{ Nondeprived smokers } \\
\hline \multicolumn{9}{|c|}{ BEGIN-END } \\
\hline & Ventral striatum & $\mathrm{R}$ & 9 & 3 & -6 & 3.18 & 18 & 0.029 \\
\hline & Anterior cingulate cortex & $L$ & -6 & 27 & 18 & 3.54 & 253 & 0.031 \\
\hline & Anterior cingulate cortex & $\mathrm{R}$ & 12 & 39 & 9 & 3.43 & 232 & 0.037 \\
\hline \multicolumn{9}{|l|}{ END-BEGIN } \\
\hline \multicolumn{9}{|c|}{ BEGIN-END } \\
\hline & Ventral tegmental area & & 3 & -18 & -21 & 3.10 & 11 & 0.009 \\
\hline & Orbitofrontal cortex & $L$ & -12 & 48 & -6 & 4.27 & 180 & 0.009 \\
\hline & Anterior cingulate cortex & L & -9 & 48 & -3 & 3.54 & 67 & 0.031 \\
\hline & Dorsolateral prefrontal cortex & L & -21 & 3 & 66 & 4.88 & 785 & 0.002 \\
\hline & Dorsolateral prefrontal cortex & $\mathrm{R}$ & 36 & -6 & 63 & 3.99 & 923 & 0.040 \\
\hline & Insular cortex & L & -36 & -3 & 12 & 3.58 & $|4|$ & 0.035 \\
\hline \multicolumn{9}{|c|}{ END-BEGIN } \\
\hline & No significant results & & & & & & & \\
\hline
\end{tabular}

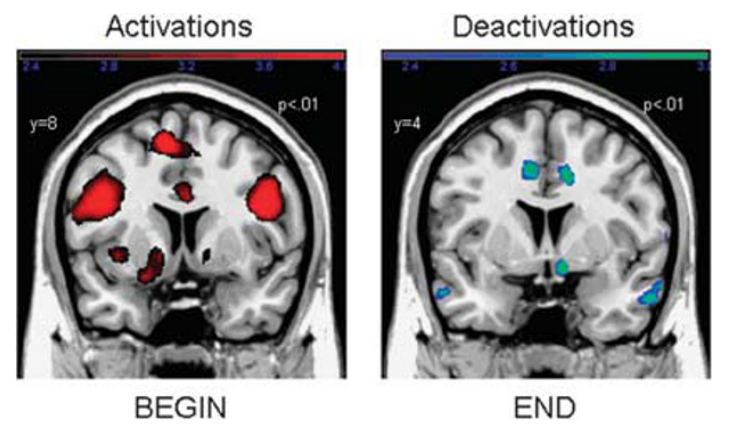

Figure 2 Neural activation and deactivation patterns for BEGIN and END in nondeprived smokers.

\section{DISCUSSION}

This study addressed two main questions: first, we wanted to explore whether stimuli associated with the beginning of smoking (BEGIN-stimuli) and stimuli associated with the terminal stage of smoking (END-stimuli) differentially activate the neural addiction network of smokers. This was on the basis of the hypothesis that the reactivity evoked by a drug-associated stimulus is critically influenced by its position in the drug-consumption ritual. Specifically, we expected BEGIN-stimuli to be excitatory as reflected in activations of the addiction network, whereas END-stimuli should have no excitatory or even inhibitory effects as reflected in deactivations. Second, we were interested in the effects of deprivation on these processes and expected an enhancement of differential effects of BEGIN- and ENDstimuli under deprivation. Additional exploratory questions addressed the issue whether other stimuli from the smoking ritual also evoke differential responses.

\section{Craving, Valence, and Arousal}

Generally, the used stimuli were rated in accordance with our hypotheses. All smoking stimuli elicited higher craving than the corresponding control stimuli in nondeprived and deprived smokers. Furthermore and in line with our previous findings (Mucha et al, 1999, 2008), in both groups BEGIN-stimuli elicited more craving than END-stimuli. Interestingly, stimuli depicting events immediately before the termination of smoking ('the last puff') also evoked more craving than END-stimuli even though they show a similar association with the peak of the nicotine effect. In contrast, stimuli depicting events immediately before the start of actual smoke consumption ('taking a cigarette out of its box') elicited less craving than BEGIN-stimuli. Overall, deprived smokers reported more craving in response to smoking stimuli than nondeprived smokers; this pattern also emerged in response to control stimuli, most probably due to craving-baseline differences (see QSU prescan scores). Differences between the craving ratings elicited by smoking $v s$ control stimuli were similar for nondeprived 
Table 6 Explorative Analysis Regarding Differences Between the Contrasts TAKING OUT, BEGIN, LAST PUFF, and END

\begin{tabular}{|c|c|c|c|c|c|c|c|c|}
\hline \multicolumn{9}{|c|}{ Nondeprived smokers vs nonsmokers } \\
\hline Main & & & & & & & & \\
\hline \multicolumn{9}{|c|}{ Group } \\
\hline & No significant results & & & & & & & \\
\hline \multicolumn{9}{|c|}{ Stage } \\
\hline \multicolumn{9}{|c|}{ Interaction } \\
\hline \multicolumn{9}{|c|}{ Group $\times$ stage } \\
\hline & Amygdala & $\mathrm{R}$ & 33 & 3 & -27 & 5.46 & 9 & 0.035 \\
\hline \multicolumn{9}{|c|}{ Deprived smokers vs nondeprived smokers } \\
\hline \multicolumn{9}{|c|}{ Stage } \\
\hline & Orbitofrontal cortex & $L$ & -12 & 51 & -3 & 8.12 & 92 & 0.028 \\
\hline & Anterior cingulate cortex & $L$ & -6 & 54 & 0 & 7.96 & 214 & 0.011 \\
\hline & Insular cortex & $\mathrm{R}$ & 39 & -3 & 3 & 7.29 & 318 & 0.028 \\
\hline \multicolumn{9}{|c|}{ Interaction } \\
\hline \multicolumn{9}{|c|}{ Group $\times$ stage } \\
\hline & Dorsolateral prefrontal cortex & $\mathrm{R}$ & 36 & -12 & 69 & 8.16 & 99 & 0.048 \\
\hline & Hippocampus & $\mathrm{R}$ & 21 & -24 & -12 & 7.89 & 72 & 0.016 \\
\hline
\end{tabular}

Two $2 \times 4$ analyses were performed, comparing nondeprived smokers with nonsmokers and deprived smokers with nondeprived smokers

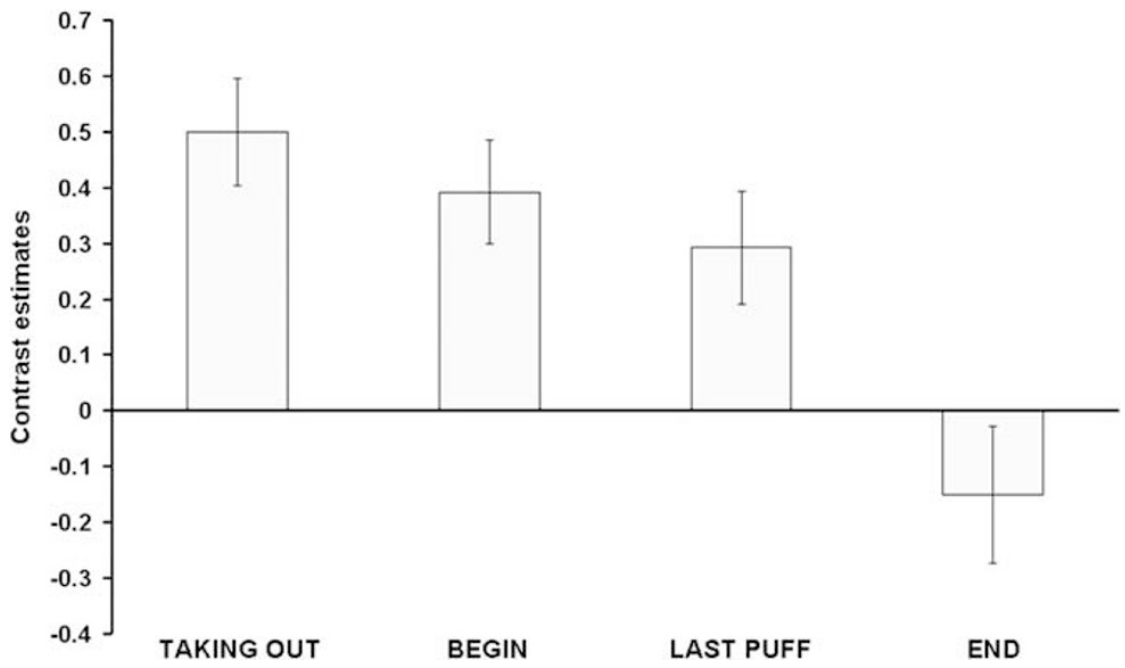

Figure 3 Main effect of stage in the ACC for nondeprived smokers and deprived smokers combined.

and deprived smokers. Thus, considering craving, we conclude that stimuli associated with different stages of the smoking ritual differentially affect craving in smokers. These effects are only slightly affected by deprivation.

A difference between BEGIN- and END-stimuli was also found for valence: similar to Mucha et al (1999, 2008), nondeprived and deprived smokers rated BEGIN-stimuli as more pleasurable than END-stimuli. At least for deprived smokers, this difference also occurred for arousal ratings. These results show that differences between BEGIN- and END-stimuli do not only exist for craving, but also for two important dimensions of emotional experience. For deprived smokers, we found that they were significantly more aroused by smoking stimuli than nondeprived smokers. This observation supports the assumption of an enhancement of the incentive value of smoking stimuli 


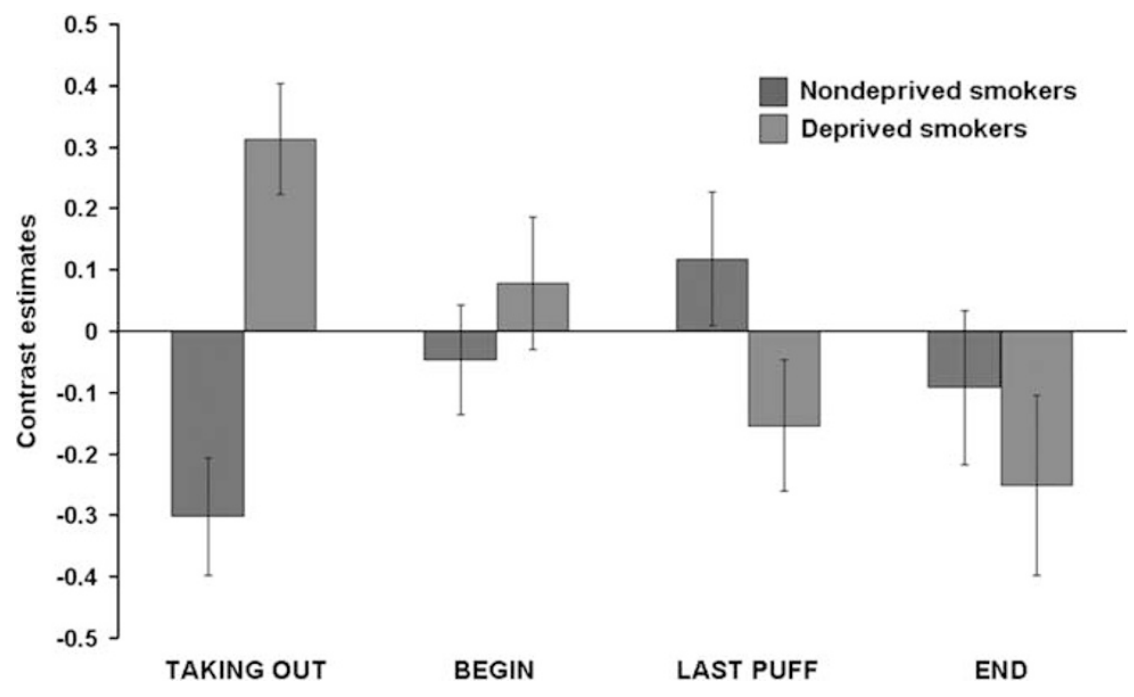

Figure 4 Group $\times$ stage interaction in the DLPFC for deprived smokers and nondeprived smokers.

under deprivation (Stewart et al, 1984, Toates, 1994; Berridge, 2004). In sum, we conclude that BEGIN- and END-stimuli lead to pronounced differences in ratings and therefore should also differentially activate the neural addiction network.

\section{Neural Impact of BEGIN- and END-Stimuli in Nondeprived Smokers}

In nondeprived smokers, prototypical BEGIN-stimuli ('lighting a cigarette') triggered activations in almost the entire addiction network. Significant stronger activations than in nonsmokers were found in the VTA, DLPFC, and the insula, three structures critically involved in reward processing, the generation and maintenance of consumption-related goals, and craving (Wilson et al, 2004; Everitt and Robbins, 2005; Naqvi and Bechara, 2009). This is in accordance with our hypothesis and resembles results obtained by others (for example, Brody et al, 2002; Due et al, 2002). In contrast, prototypical END-stimuli ('stubbing out a cigarette butt') led to a more complex pattern of activations and deactivations. Importantly, in nondeprived smokers, deactivations in response to END-stimuli were found in parts of the reward and the control subcircuit: ventral striatum and ACC. Contrasting BEGIN- and ENDstimuli, we also found higher neural activity toward BEGINstimuli in these two structures. This shows that END-stimuli have indeed a differential impact on the neuronal activity in the addiction network of nondeprived smokers. As the ventral striatum and the ACC have important roles for addiction in terms of incentive motivation, reward detection, and disinhibition (for example, Robinson and Berridge, 1993, 2003; Volkow et al, 2003, 2004; Lubman et al, 2004), the observed deactivations in these structures may indicate inhibitory effects of prototypical END-stimuli.

The role of the ventral striatum in reward processing and reward expectancy is widely acknowledged (for example, Spanagel and Weiss, 1999; Wise, 2004) and its activity has been shown to be correlated with the magnitude of a received reward (Knutson et al, 2001, 2005). Moreover, unexpected omissions of a reward as well as stimuli predictive of reward omissions (that is, a conditioned inhibitor) lead to an inhibition of dopaminergic neurons (Schultz, 1998, 2002; Tobler et al, 2003). Furthermore, previous behavioral studies show that stimuli predicting the absence of drug can suppress drug seeking (Kearns et al, 2005). Therefore, the deactivations observed in our study might point to a reduction of an incentive motivational state in response to prototypical END-stimuli in smokers.

The ACC is a part of the limbic affect-response system (Wexler et al, 2001) and interconnected with the ventral striatum (Vogt et al, 1992; Devinsky et al, 1995; Bush et al, 2000). In addicts, it is one of the structures, in which activity in response to stimuli paired with a variety of drugs, such as nicotine (Brody et al, 2002; Due et al, 2002; McClernon et al, 2005; McBride et al, 2006), cocaine (Maas et al, 1998; Childress et al, 1999; Garavan et al, 2000; Kilts et al, 2001; Wexler et al, 2001), and heroin (Daglish et al, 2001), is most frequently reported. Moreover, its importance for addiction is emphasized by the fact that surgical lesioning of the ACC can decrease drug consumption in addicts (Sharma, 1974; Kanaka and Balasubramaniam, 1978). Likewise, smokers treated with bupropion showed an attenuation of both, cueinduced cigarette craving and ACC activation (Brody et al, 2004). Like the ventral striatum, the ACC is involved in reward processing and it also seems to be sensitive to the magnitude of a received reward (Bush et al, 2002; Peoples, 2002; Shidara and Richmond, 2002; Kirsch et al, 2003). It has also been shown to have a role in anticipation (Murtha et al, 1996). In conditioning studies, the ACC was found to discriminate between a reinforced CS $(\mathrm{CS}+)$ and a nonreinforced CS (CS-) (Martin-Soelch et al, 2007). Therefore, the differential activation of the ACC by BEGIN- and END-stimuli could be due to different anticipatory states, resulting from the discrimination of stimuli with different predictive values. Finally, the ACC has a role in the detection of conflicts (Braver et al, 2001) and thus is activated in tasks that generate conflicting response tendencies (for example, Carter et al, 1999). As Wexler (2001) notes, subjects in cue reactivity paradigms may be faced with an internal response conflict and the need to inhibit certain response tendencies. This also 
could contribute to our data, with very pronounced conflicts in response to BEGIN-stimuli but not in response to END-stimuli.

Surprisingly, although these differences between BEGINand END-stimuli were significant in nondeprived smokers but not in nonsmokers, there were no significant differences between both groups. This could possibly be due to a motivational impact of smoking stimuli on nonsmokers. For example, BEGIN-stimuli could be aversive because they are associated with smoke; END-stimuli might have a relieving effect. The ventral striatum and ACC are known to be involved in a wide range of positive as well as negative emotional processes (Rolls, 1999; Martin-Soelch, 2007). Thus, although stimuli can have different motivational impacts, they can lead to activations in the same brain structures.

\section{Neural Impact of BEGIN- and END-Stimuli in Deprived Smokers}

The comparison between deprived smokers and nondeprived smokers revealed some interesting results. Regarding BEGIN-stimuli ('lighting a cigarette'), we expected that deprived smokers would show enhanced neuronal activity compared with nondeprived smokers. However, both groups barely differed (see also Geier et $a l, 2000$ and Mueller et al, 1998 for similar results regarding the modulation of the startle response). Regarding END-stimuli ('stubbing out a cigarette butt'), we also expected enhanced effects under deprivation, that is, lower responses in deprived smokers compared with nondeprived smokers. Indeed, we observed lower responses in the OFC, DLPFC, and insula in deprived smokers compared with nondeprived smokers. Moreover, we found deactivations as well as activations in the OFC and exclusive deactivations in the DLPFC of deprived smokers. These structures are associated with craving, drug expectancy, and planning of drug consumption (Goldstein and Volkow, 2002; Wilson et al, 2004; Naqvi and Bechara, 2009). A review regarding the role of drug expectancy on neuronal responses to drug cues (Wilson et al, 2004) found that only active drug users showed OFC and DLPFC activations in response to cues. Drug users in treatment, who are supposed to have no expectancy of their drug in response to cues, showed no such activations. This was further confirmed by McBride et al (2006) who explicitly manipulated the expectancy to smoke after a cue exposure experiment. Similar effects could account for our data. One essential feature of ENDstimuli may be that the perceived availability of smoke is reduced (Mucha et al, 2008). This could lead to a lower drug expectancy and less planning of drug consumption, especially in deprived smokers. Overall, deprivation particularly seems to affect the responses to END-stimuli, whereas responses to BEGIN-stimuli seem to be only slightly affected. As a consequence, we found significantly greater differences between BEGIN- and END-stimuli in deprived smokers than in nondeprived smokers in OFC and DLPFC.

Thus, deprivation does not simply enhance responses to all drug stimuli. Rather, the effect of deprivation strongly depends on the stage of the consumption ritual a smoking stimulus is associated with.

\section{Neural Impact of Additional Stimuli}

Two additional stimuli were included in our study, 'taking a cigarette out of its box' and 'the last puff. In nondeprived smokers, 'taking a cigarette out of its box' led to a complex pattern of activations and deactivations. Activations as well as deactivations occurred in the reward and control subcircuits (ventral striatum, ACC, DLPFC). 'The last puff led to activations in the entire network. Surprisingly, although 'the last puff directly precedes the prototypical END-stimuli, neural responses are more similar to those of prototypical BEGIN-stimuli. Likewise, 'taking a cigarette out of its box' directly precedes prototypical BEGINstimuli, but the results are more similar to those of prototypical END-stimuli.

Comparing nondeprived smokers and nonsmokers (group $\times$ stage analysis), we found stronger amygdala activation to 'the last puff in nondeprived smokers and to BEGIN-stimuli in nonsmokers. Among other functions, the amygdala is involved in the facilitation of attention to salient events and processes stimuli predictive of aversive as well as positive events (LeDoux, 2000; Baxter and Murray, 2002; Phelps and LeDoux, 2005). In addiction research, amygdala activity is related to the processing of discrete cues (for example, Everitt and Robbins, 2005). The activation in nonsmokers emphasizes the motivational significance of BEGIN-stimuli in this group as mentioned before. The activation in nondeprived smokers suggests 'the last puff to be a very salient stimulus for this group. This might be explained by the fact that it displays acute smoke availability and intake. In addition, nicotine intake might be higher with 'the last puff' due to the reduced air resistance of a cigarette butt. 'Taking a cigarette out of its box' and BEGIN-stimuli predict a rather prospective nicotine availability. In a nondeprived state, this may not be as salient as 'the last puff.

In the group $\times$ stage analysis for deprived smokers and nondeprived smokers, a significant main effect for the factor stage occurred in the ACC, OFC, and the insula. OFC and ACC responses diminished over the time course of smoking, leading to pronounced deactivations in response to END-stimuli. The role of the ACC in addiction was discussed above. The OFC is linked to similar functions, particular to craving and to the expectation of drug (Goldstein and Volkow, 2002). This again shows that END-stimuli seem to yield a unique response in the addiction network that is different from prototypical BEGIN-stimuli and other stimuli of the smoking ritual. In this analysis, deactivations to END-stimuli have also been found in the insula. The role of the insula in addiction has only recently been emphasized (Naqvi and Bechara, 2009), and similar to the OFC, it has become linked to craving. However, the OFC seems to be more involved in the cognitive aspects of craving like drug expectation (London et al, 2000; Goldstein and Volkow, 2002; see also Cardinal et al, 2002). The insula, owing to its function in monitoring physiological processes (Craig, 2009; Naqvi and Bechara, 2009), seems to be more involved in the bodily aspects. Furthermore, significant interactions in the DLPFC and hippocampus show a remarkable difference between deprived and nondeprived smokers. As mentioned above, the DLPFC is crucially involved in the generation and 
maintenance of consume-related goals. Its activity is thereby modulated by the perceived availability to consume the drug (Wilson et al, 2004). In deprived smokers, neuronal activity in the DLPFC is highest in response to 'taking a cigarette out of its box' and diminishes over time. 'The last puff and END-stimuli are accompanied with deactivations of DLPFC activity. We therefore conclude that in a deprived state the most salient smoking stimuli are those that predict more prospective drug availability. As noted above, in a nondeprived, that is, satiated state, stimuli predicting rather acute drug availability seem to be the most salient cues. This can also be seen in the DLPFC activity of nondeprived smokers that is deactivated in response to 'taking a cigarette out of its box', BEGIN-stimuli, and ENDstimuli. Only 'the last puff leads to strong DLPFC activations in this group. It is well known that drug availability has an important role in the modulation of cue reactivity (Carter and Tiffany, 2001; Wertz and Sayette, 2001; Wilson et al, 2004; McBride et al, 2006). Furthermore, Mucha et al (2008) suggested that the perceived smoke availability is an important factor for the differential reactivity to BEGIN- and END-stimuli. Thus, on a neural level, our results seem to confirm their findings but also lead to a possible extension: in nondeprived smokers particularly stimuli depicting the acute availability of smoke lead to activations in the addiction network. In contrast, in a deprived state mainly stimuli predicting a prospective availability lead to the strongest activations. Similar effects were found in a study on food deprivation (Siep et al, 2009); subjects saw pictures of high- or low-calorie food while being either hungry or satiated. The results revealed an increased reward processing in response to low-calorie food in satiated subjects but in response to high calorie food in deprived subjects.

Previous findings regarding the effects of drug deprivation on subjective (Gross et al, 1993; Mucha et al, 1999; Waters and Feyerabend, 2000), physiological (Geier et al, 2000), and neuronal responses are mixed. For neuronal activity, deprived smokers (compared with nondeprived smokers) have been found to show weaker (David et al, 2007), equal (McBride et al, 2006; McClernon et al, 2005), or greater activity (McClernon et al, 2008) in response to smoking cues. Yet, these studies did not differentiate their stimuli regarding to the position in the smoking ritual and the perceived acute or prospective drug availability. This might be one factor that can explain differences between studies. However, we have to note that these brain data are not fully in accordance with subjective data. For example, both, nondeprived smokers and deprived smokers gave higher craving ratings in response to BEGIN-stimuli than in response to the other smoking stimuli.

\section{Limitations}

This study was the first investigation of neuronal responses to stimuli related to different stages of the smoking ritual. Careful attention was paid to the development of the stimulus and control material and the composition of three participant groups: nonsmokers, nondeprived smokers, and deprived smokers. However, some limitations may lie in possible habituation effects developing over the experimental course. Further, an unwanted transfer effect because of the random arrangement of the different stages of the smoking ritual, which normally has a strictly determined sequence, might have occurred. Eventually, we were able to show some deprivation effects, yet more pronounced effects might have been observed with a longer deprivation period.

\section{Conclusions}

Our results support the notion that stimuli depicting the terminal stage of the smoking ritual (END-stimuli) modulate neuronal activity in the addiction network of smokers in a different way than stimuli depicting the beginning of the smoking ritual (BEGIN-stimuli). BEGIN-stimuli lead to significantly stronger activations than END-stimuli and, furthermore, END-stimuli lead to partial deactivations in the network. As expected, deprivation leads to a stronger differentiation between BEGIN- and END-stimuli in deprived than in nondeprived smokers. Furthermore, activity in response to END-stimuli is significantly lower in deprived smokers, whereas responses to BEGIN-stimuli differ only marginally. Deprived smokers seem to respond particularly strong to stimuli signaling prospective drug availability, whereas nondeprived smokers respond particularly strong to stimuli signaling acute drug availability.

\section{ACKNOWLEDGEMENTS}

The study was supported by the German Research Foundation (DFG): Forschergruppe 'Emotion and Behaviour' (STA475/5-1) and Graduiertenkolleg 'NeuroAct'.

\section{DISCLOSURE}

Ronald F Mucha has invested in a marketing venture to use pictures of the end of smoking for symbolizing smoking with little craving. This could be perceived as a conflict of interest, although this study was conceived and carried out independent of this. The remaining authors do not have any conflict of interest, financial or otherwise, which might bias this work.

\section{REFERENCES}

Baxter MG, Murray EA (2002). The amygdala and reward. Nat Rev Neurosci 3: 563-573.

Berridge KC (2004). Motivation concepts in behavioral neuroscience. Physiol Behav 81: 179-209.

Bradley MM, Lang PJ (1994). Measuring emotion: the selfassessment manikin and the semantic differential. J Behav Ther Exp Psychiatry 25: 49-59.

Braver TS, Barch DM, Gray JR, Molfese DL, Snyder A (2001). Anterior cingulate cortex and response conflict: effects of frequency, inhibition and errors. Cerebral Cortex 11: 825-836.

Brody AL, Mandelkern MA, London ED, Childress AR, Lee GS, Bota RG et al (2002). Brain metabolic changes during cigarette craving. Arch Gen Psychiatry 59: 1162-1172.

Brody AL, Mandelkern MA, Lee G, Smith E, Sadeghi M, Saxena S (2004). Attenuation of cue-induced cigarette craving and anterior cingulate cortex activation in bupropion-treated smokers: a preliminary study. Psychiatry Res: Neuroimaging 130: 269-281. 
Bush G, Luu P, Posner MI (2000). Cognitive and emotional influences in anterior cingulate cortex. Trends Cogn Sci 4: 215222.

Bush G, Vogt BA, Holmes J, Dale AM, Greve D, Jenike MA et al (2002). Dorsal anterior cingulate cortex: a role in reward-based decision making. Proc Natl Acad Sci USA 99: 523-528.

Bushnell PJ, Levin ED, Marrocco RT, Sarter MF, Strupp BJ, Warburton DM (2000). Attention as a target of intoxication: insights and methods from studies of drug abuse. Neurotoxicol Teratol 22: 487-502.

Cardinal RN, Parkinson JA, Hall J, Everitt BJ (2002). Emotion and motivation: the role of the amygdala, ventral striatum, and prefrontal cortex. Neurosci Biobehav Rev 26: 321-352.

Carter BL, Tiffany ST (1999). Meta-analysis of cue-reactivity in addiction research. Addiction 94: 327-340.

Carter BL, Tiffany ST (2001). The cue-availability paradigm: the effects of cigarette availability on cue reactivity in smokers. Exp Clin Psychopharmacol 9: 183-190.

Carter CS, Botvinick MM, Cohen JD (1999). The contribution of the anterior cingulate cortex to executive processes in cognition. Rev Neurosci 10: 49-57.

Childress AR, Mozley PD, McElgin W, Fitzgerald J, Reivich M, O'Brien CP (1999). Limbic activation during cue-induced cocaine craving. Am J Psychiatry 156: 11-18.

Craig AD (2009). How do you feel-now? The anterior insula and human awareness. Nat Rev Neurosc 10: 59-70.

Daglish MRC, Weinstein A, Malizia AL, Wilson S, Melichar JK, Britten S et al (2001). Changes in regional cerebral blood flow elicited by craving memories in abstinent opiate-dependent subjects. Am J Psychiatry 158: 1680-1686.

David SP, Munafò MR, Johansen-Berg H, Smith SM, Rogers RD, Matthews PM et al (2005). Ventral striatum/nucleus accumbens activation to smoking-related pictorial cues in smokers and nonsmokers: a functional magnetic resonance imaging study. Biol Psychiatry 58: 488-494.

David SP, Munafò MR, Johansen-Berg H, MacKillop J, Sweet LH, Cohen RA et al (2007). Effects of acute nicotine abstinence on cue-elicited ventral striatum/nucleus accumbens activation in female cigarette smokers: a functional magnetic resonance imaging study. Brain Imaging Behav 1: 43-57.

Della Casa V, Höfer I, Weiner I, Feldon J (1999). Effects of smoking status and schizotypy on latent inhibition. J Psychopharmacol 13: 45-57.

Devinsky O, Morrell MJ, Vogt BA (1995). Contributions of anterior cingulate cortex to behaviour. Brain 118: 279-306.

Due DL, Huettel SA, Hall WG, Rubin DC (2002). Activation in mesolimbic and visuospatial neural circuits elicited by smoking cues: evidence for functional magnetic resonance imaging. Am J Psychiatry 159: 954-960.

Everitt BJ, Dickinson A, Robbins TW (2001). The neuropsychological basis of addictive behaviour. Brain Res Rev 36: 129-138.

Everitt BJ, Robbins TW (2005). Neural systems of reinforcement for drug addiction: from actions to habits to compulsion. Nat Neurosci 8: 1481-1489.

Franklin TR, Wang Z, Wang J, Sciortino N, Harper D, Li Y et al (2007). Limbic activation to cigarette smoking cues independent of nicotine withdrawal: a perfusion fMRI study. Neuropsychopharmacology 32: 2301-2309.

Garavan H, Pankiewicz J, Bloom A, Cho JK, Sperry L, Ross TJ et al (2000). Cue-induced cocaine craving: neuroanatomical specificity for drug users and drug stimuli. Am J Psychiatry 157: 17891798.

Geier A, Mucha RF, Pauli P (2000). Appetitive nature of drug cues confirmed with physiological measures in a model using pictures of smoking. Psychopharmacology 150: 283-291.

Goldstein RZ, Volkow ND (2002). Drug addiction and its underlying neurobiological basis: neuroimaging evidence for the involvement of the frontal cortex. Am J Psychiatry 159: 1642-1652.
Gross TM, Jarvik ME, Rosenblatt MR (1993). Nicotine abstinence produces content-specific stroop interference. Psychopharmacology 110: 333-336.

Hyman SE (2005). Addiction: a disease of learning and memory. American Journal of Psychiatry 162: 1414-1422.

Kalivas PW, Volkow ND (2005). The neural basis of addiction: a pathology of motivation and choice. Am J Psychiatry 162: 1403-1413.

Kanaka TS, Balasubramaniam V (1978). Stereotactic cingulotomy for drug addiction. Applied Neurophysiology 41: 86-92.

Kauer JA, Malenka RC (2007). Synaptic plasticity and addiction. Nat Rev Neurosci 8: 844-858.

Kearns DN, Weiss SJ, Schindler CW, Panlilio LV (2005). Conditioned inhibition of cocaine seeking in rats. J Exp Psychol 31: 247-253.

Kilts CD, Schweitzer JB, Quinn CK, Gross RE, Faber TL, Muhammad $\mathrm{F}$ et al (2001). Neural activity related to drug craving in cocaine addiction. Arch Gen Psychiatry 58: 334-341.

Kirsch P, Schienle A, Stark R, Sammer G, Blecker C, Walter B et al (2003). Anticipation of reward in a nonaversive differential conditioning paradigm and the brain reward system: an eventrelated fMRI study. Neuroimage 20: 1086-1095.

Knutson B, Adams CA, Fong GW, Hommer D (2001). Anticipation of increasing monetary reward selectively recruits nucleus accumbens. J Neurosci 21: 1-5.

Knutson B, Taylor J, Kaufman M, Peterson R, Glover G (2005). Distributed neural representation of expected value. J Neurosci 25: 4806-4812.

Lang PJ, Bradley MM, Cuthbert B (1995). International Affective Picture System. Center for Research in Psychophysiology, University of Florida: Gainsville, Florida.

LeDoux JE (2000). Emotion circuits in the brain. Annu Rev Neurosci 23: 155-184.

London ED, Ernst M, Grant S, Bonson K, Weinstein A (2000). Orbitofrontal cortex and human drug abuse: functional imaging. Cerebral Cortex 10: 334-342.

Lubman DJ, Yücel M, Pantelis C (2004). Addiction, a condition of compulsive behaviour? Neuroimaging and neuropsychological evidence of inhibitory dysregulation. Addiction 99: 1491-1502.

Maas LC, Lukas SE, Kaufman MJ, Weiss RD, Daniels SL, Rogers VW et al (1998). Functional magnetic resonance imaging of human brain activation during cue-induced cocaine craving. Am J Psychiatry 155: 124-126.

Martin-Soelch C, Linthicum J, Ernst M (2007). Appetitive conditioning: neural bases and implications for psychopathology. Neurosci Biobehav Rev 31: 426-440.

McBride D, Barret SP, Kelly JT, Aw A, Dagher A (2006). Effects of expectancy and abstinence on the neural response to smoking cues in cigarette smokers: an fMRI study. Neuropsychopharmacology 31: 2728-2738.

McClernon FJ, Hiott FB, Huettel SA, Rose JE (2005). Abstinenceinduced changes in self-report craving correlate with eventrelated fMRI responses to smoking cues. Neuropsychopharmacology 30: 1940-1947.

McClernon FJ, Kozink RV, Lutz AM, Rose JE (2008). 24-h smoking abstinence potentiates fMRI-BOLD activation to smoking cues in cerebral cortex and dorsal striatum. Psychopharmacology 204: 25-35.

Mucha RF, Geier A, Pauli P (1999). Modulation of craving by cues having differential overlap with pharmacological effect: evidence for cue approach in smokers and social drinkers. Psychopharmacology 147: 113-306.

Mucha RF, Pauli P, Weyers P (2006). Psychophysiology and implicit cognition in drug use: significance and measurement of motivation for drug use with emphasis on startle tests. In: Wiers RW, Stacy AW (eds). Handbook of Implicit Cognition and Addiction. Sage Publications: Thousand Oaks. pp 201-214. 
Mucha RF, Pauli P, Weber M, Winkler M (2008). Smoking stimuli from the terminal phase of cigarette consumption may not be cues for smoking in healthy smokers. Psychopharmacology 201: 81-95.

Mueller V, Mucha RF, Pauli P (1998). Dependence on smoking and the acoustic startle response in healthy smokers. Pharmacol Biochem Behav 59: 1031-1038.

Müller V, Mucha RF, Ackermann K, Pauli P (2001). Die Erfassung des Cravings bei Rauchern mit einer deutschen Version des 'Questionnaire on Smoking Urges' (QSU-G). Zeitschrift für Klinische Psychologie und Psychotherapie 30: 164-171.

Murtha S, Chertkow H, Beauregard M, Dixon R, Evans A (1996). Anticipation causes increased blood flow to the anterior cingulate cortex. Hum Brain Mapp 4: 103-112.

Naqvi NH, Bechara A (2009). The hidden island of addiction: the insula. Trends Neurosci 32: 56-67.

Niaura RS, Rohsenow DJ, Binkoff JA, Monti PM, Pedraza M, Abrams DB (1988). Relevance of cue reactivity to understanding alcohol and smoking relapse. J Abnormal Psychol 97: 133-152.

O'Brien CP, Childress AR, Ehrman R, Robbins SJ (1998). Conditioning factors in drug abuse: can they explain compulsion? J Psychopharmacol 12: 15-22.

Oldfield R (1971). The assessment and analysis of handedness: the Edingburgh Inventory. Neuropsychologia 9: 97-113.

Panlilio LV, Thorndike EB, Schindler CW (2008). A stimuluscontrol account of regulated drug intake in rats. Psychopharmacology 196: 441-450.

Peoples L (2002). Will, anterior cingulate cortex, and addiction. Science 296: 1623-1624.

Phelps EA, LeDoux JE (2005). Contributions of the amygdala to emotion processing: from animal models to human behaviour. Neuron 48: 175-187.

Robinson TE, Berridge KC (1993). The neural basis of drug craving: an incentive-sensitization theory of addiction. Brain Res Brain Res Rev 18: 247-291.

Robinson TE, Berridge KC (2003). Addiction. Annu Rev Psychol 54: 25-53.

Rolls ET (1999). The Brain and Emotion.. Oxford University Press: New York.

Schultz W (1998). Predictive reward signal of dopamine neurons. $J$ Neurophysiol 80: 1-27.

Schultz W (2002). Getting formal with dopamine and reward. Neuron 36: 241-263.

Schumann A, Rumpf HJ, Meyer C, Hapke U, John U (2003a). Deutsche Version des Fagerström-Test for Nicotine Dependence (FTND-G) und des Heaviness of Smoking Index (HIS-G). In Glöckner-Rist A, Rist F, Küfner H (eds). Elektronisches Handbuch zu Erhebungsinstrumenten im Suchtbereich (EHES) Version 300 Zentrum für Umfragen, Methoden und Analysen: Mannheim.

Self DW, Nestler EJ (1998). Relapse to drug-seeking: neural and molecular mechanisms. Drug Alcohol Depend 51: 49-60.

Sharma T (1974). Abolition of opiate hunger in humans following bilateral anterior cingulotomy. Tex Med 70: 49-52.

Shidara M, Richmond BJ (2002). Anterior cingulate: single neuronal signals related to degree of reward expectancy. Science 296: 1709-1711.

Siegel S (1989). Pharmacological conditioning and drug effects. In: Goudie AJ, Emmet-Oglesby MW (eds). Psychoactive Drugs: Tolerance and Sensitization. Humana Press: Clifton, NJ. pp 115-180.
Siep N, Roefs A, Roebroeck A, Havermans R, Bonte ML, Jansen A (2009). Hunger is the best spice: an fMRI study of the effects of attention, hunger and calorie content on food rewards processing in the amygdala and orbitofrontal cortex. Behav Brain Res 198: $149-158$

Silva FJ, Timberlake W, Koehler TL (1996). A behavior systems approach to bidirectional excitatory conditioning. Learn Motiv 27: $130-150$.

Silva KM, Timberlake W (1997). A behavior systems view of conditioned states during long and short CS-US intervals. Learn Motiv 28: 465-490.

Silva FJ, Timberlake W, Cevik MO (1998). A behaviour systems approach to the expression of backward associations. Learn Motiv 29: 1-22.

Smolka MN, Bühler M, Klein S, Zimmermann U, Mann K, Heinz A et al (2006). Severity of nicotine dependence modulates cueinduced brain activity in regions involved in motor preparation and imagery. Psychopharmacology 184: 577-588.

Spanagel R, Weiss F (1999). The dopamine hypothesis of reward: past and current status. Trends Neurosci 22: 521-527.

Stewart J, de Wit H, Eikelboom R (1984). Role of conditioned and unconditioned drug effects in the self-administration of opiates and stimulants. Psychol Rev 91: 251-268.

Toates F (1994). Comparing motivational systems - an incentive motivation perspective (1994). In: Booth DA, Legg CR (eds). Appetite: Neural and Behavioural Bases. Oxford University Press: London. pp 305-327.

Tobler PN, Dickinson A, Schultz W (2003). Coding of predicted reward omission by dopamine neurons in a conditioned inhibition paradigm. J Neurosci 23: 10402-10410.

Timberlake W (1997). An animal-centered, causal-system approach to the understanding and control of bahvior. Appl Anim Behav Sci 53: 107-129.

Vogt BA, Finch DM, Olson CR (1992). Functional heterogeneity in cingulate cortex: the anterior executive and posterior evaluative regions. Cerebral Cortex 2: 435-443.

Volkow ND, Fowler JS, Wang GJ (2003). The addicted human brain: insights from imaging studies. J Clin Investig 111: 14441451.

Volkow ND, Fowler JS, Wang GJ (2004). The addicted human brain viewed in the light of imaging studies: brain circuits and treatmet strategies. Neuropharmacology 47: 3-13.

Waters AJ, Feyerabend C (2000). Determinants and effects of attentional bias in smokers. Psychol Addict Behav 14: 111-120.

Wertz JM, Sayette MA (2001). A review of the effects of perceived drug use opportunity on self-reported urge. Exp Clin Psychopharmacol 9: 3-13.

Wexler BE, Gottschalk CH, Fulbright RK, Prohovnik I, Lacadie CM, Rounsaville BJ et al (2001). Functional magnetic resonance imaging of cocaine craving. Am J Psychiatry 158: 86-95.

Wikler A (1948). Recent progress in research on the neurophysiologic basis of morphine addiction. Am J Psychiatry 105: 329338.

Wilson SJ, Sayette MA, Fiez JA (2004). Prefrontal responses to drug cues: a neurocognitive analysis. Nat Neurosci 7: 211-214.

Wise RA (2004). Dopamine, learning and motivation. Nat Rev Neurosci 5: 1-12.

Worsley KJ (2001). Statistical analysis of activation images. In Jezzard P, Matthews PM, Smith SM (eds). Functional MRI: An Introduction to methods. Oxford University Press: Oxford. pp 251-270. 